\title{
Estrategias motivadoras en pruebas escritas para mejorar el aprendizaje de la Programación de Computadores
}

\author{
A strategy in written exams to promote students to learning computer \\ programming
}

O. I. Trejos-Buriticá iD

\begin{abstract}
Resumen-El propósito investigativo que inspira este artículo es comparar el impacto de tres estrategias motivadoras en evaluaciones escritas frente al método de la evaluación tradicional en Programación de Computadores. Se acudió a un estudio durante seis semestres con grupos de una misma asignatura. Cada grupo se dividió en dos subgrupos; con uno de ellos se aplicó la metodología presentada y con el otro se aplicó la forma tradicional de evaluación escrita, todo ello apoyado en características fisiológicas del cerebro, la manera como aprende el cerebro y la estrategia Brain Based Learning. Los resultados evidencian que cuando el cerebro se encuentra en momentos de tensión, una acción bien conducida permite que elementos clave de aprendizaje se ubiquen en la memoria a largo plazo. Se concluye que, si se tienen claros los objetivos de una evaluación y su diseño es apropiado, conviene aplicar estas estrategias en las evaluaciones escritas tanto en programación de computadores como en otras áreas disciplinares. Se ha adoptó un enfoque cuantitativo asociado a un estudio de caso grupal.
\end{abstract}

Palabras clave - Aprendizaje a través de la experiencia, aprendizaje continuo, evaluación de conocimientos anteriores, motivación, programación informática.

Abstract-The purpose of the research that inspires the present article is to compare the impact of three motivating strategies during written evaluations versus the traditional evaluation in Computer Programming. We went to a comparative study over six semesters with groups of the same subject. Each group was divided into two subgroups, with one of them applying the methodology proposed in this article and with the other we use the traditional form of written evaluation, all supported by physiological characteristics of the brain, the way the brain learns and the Brain Based Learning strategy. The results show that when the brain is in moments of academic stress, a well-conducted action can allow key elements of learning to be located in the long term memory. It is concluded that it is worth knowing these three strategies to apply them in written evaluations in other disciplinary areas and, once the objectives of the evaluation have been clearly identified and it has been properly designed, the knowledge is located in the memory long term.

Index Terms - Brain based learning, Computer programming, Motivation, Systems Engeneering, Written exams

Este manuscrito fue enviado el 19 de marzo de 2019 y aceptado el 26 de diciembre de 2019. El presente artículo es un subproducto del proyecto de investigación Código 6-16-13 "Desarrollo de un modelo metodológico para el aprendizaje de la programación imperativa en Ingeniería de Sistemas basado en aprendizaje significativo, aprendizaje por descubrimiento y el modelo 4Q de

\section{INTRODUCCIÓN}

Según la teoría de aprendizaje significativo, dos factores son de gran importancia en el proceso de aprendizaje: lo que el alumno ya sabe [1] y la motivación que tenga para aprender [2] debido a que el primero podría interpretarse como una mirada hacia atrás para tener consciencia de lo que se tiene en la base cognitiva y el segundo es un provocación hacia adelante para anhelar actualizar, modificar, mejorar o desplazar el conocimiento que ya se ha adquirido por otro más acorde con el contexto que rodea al ser humano. La motivación se constituye en la manera como un estudiante vive su deseo de aprender [3] y lo expresa a través de su participación en actividades y estrategias, todo ello en pro de adquirir nuevos conocimientos y confrontarlos con los conocimientos ya adquiridos.

El propósito de la presente investigación radica en comparar lo que sucede con los estudiantes cuando, inmersos en los estados de tensión naturales que provoca una prueba escrita, se les abren espacios de motivación inesperados, cronometrados y calculados (dentro de la misma prueba) para que el cerebro capte aquel conocimiento que le hace falta para resolver una situación, un ejercicio o un enunciado. El objetivo de la comparación radica en confrontar la metodología expuesta frente a la metodología tradicional de evaluaciones escritas en la cual se le entrega a un estudiante un conjunto de enunciados y ejercicios para que sean resueltos en el tiempo que provee la sesión de clase y, luego de pasado el tiempo destinado para ello, se les recoge finalizando con ello la realización de la misma.

El problema a abordar radica en que la responsabilidad de la motivación del aprendizaje va de la mano del docente que conduzca el proceso como tal y se ha querido cuestionar si dicha motivación, manifestada en los términos explicados, mejora el aprendizaje de los estudiantes de programación de computadores o si, definitivamente, logra los mismos objetivos que logra la metodología tradicional. Para ello debe tenerse en cuenta que Los seres humanos tenemos enorme dificultad para

preferencias de pensamiento" aprobado por la Vicerrectoría de Investigaciones, Innovación y Extensión de la Universidad Tecnológica de Pereira.

Omar Iván Trejos Buriticá, Docente de Planta, Ingeniería de Sistemas y Computación, categoría Titular, Universidad Tecnológica de Pereira, omartrejos@utp.edu.co 
transformar procesos que vienen desarrollándose en una dirección y de una manera determinada [4].

La novedad de la metodología investigada y probada en el aula radica en que durante el lapso de tiempo destinado para las pruebas escritas se abren puertas que, tradicionalmente, han estado cerradas a la investigación educativa y que, según lo determinen los resultados, puedan ser grandes disparadores del aprendizaje y motivadores excelsos de los cambios que requiere la base cognitiva cuando un nuevo conocimiento se confronta con un conocimiento previo. Por tal motivo se parte de que existe un acuerdo de que los elementos configuradores de este tiempo-espacio actual son: el conocimiento, la tecnología, los nuevos lenguajes, la información, la comunicación, la innovación y la investigación [5].

Se justifica este estudio porque se hace necesario indagar, allende los avances tecnológicos y científicos, acerca de cómo se puede avanzar pedagógicamente para que el aprendizaje, como proceso de apropiación, asimilación, aplicación, retroalimentación y evaluación del conocimiento, sea la base para el avance de lo que ya se sabe por caminos que exijan menos esfuerzos y mayor efectividad. De la misma forma, es claro que en la medida en que se encuentren caminos ágiles para el aprendizaje de cualquier nuevo conocimiento, se podrá avanzar mucho más en la profundización del mismo ya que la educación también puede alentar a un sentido general de la espiritualidad, es decir, de la perspectiva a estar abierto a formas que superan lo meramente instrumental pues hasta ahora se ha limitado a una acción memorística de contenidos muchas veces irrelevantes y alejados de la vida [6] sin desconocer que se hace necesario articular a los docentes con nuevas formas de comunicación, con nuevas formas de lectura y nuevas relaciones que surgen entre los estudiantes y entre ellos y los profesores [7].

Para el desarrollo del presente artículo se ha acudido a literatura especializada cuyo corpus presenta algunas teorías como la de aprendizaje significativo y aprendizaje por descubrimiento así como estrategias como Brain Based Learning, características del cerebro y la manera como aprende el ser humano desde la perspectiva neurológica. De otra parte se han recolectado resultados obtenidos de la aplicación de esta metodología en los semestres I y II de los años 2016, 2017 y 2018 en cursos de programación de computadores del programa Ingeniería de Sistemas y Computación de la Universidad Tecnológica de Pereira. Para la construcción de este artículo se ha adoptado la redacción científica como fondo y la forma se ha tomado de la estructura IMRYD según la cual se ha presenta una introducción seguida de un marco teórico, luego se explica la metodología utilizada junto con sus resultados y por último se realiza una discusión analítica que finaliza con unas conclusiones. Las referencias bibliográficas al final son el respaldo del conocimiento científico planteado a lo largo de la investigación.

\section{MARCO TEÓRICO}

La motivación se convierte en el motor que facilita el camino para que se modifique la base cognitiva del ser humano y se puedan adquirir nuevos conocimientos con una perdurabilidad que se aproxime al mediano y largo plazo [8]. La motivación se define como la voluntad indeclinable de querer aprender por parte del estudiante y de saber superar las vicisitudes que se encontraren en el camino de dicho proceso de aprendizaje [9]. Gran parte de la motivación, como mecanismo que impulsa el aprendizaje, recae sobre el docente quien debe tener las herramientas pedagógicas y el conocimiento disciplinar para que los estudiantes quieran saber más de su área específica y en donde dicho docente se convierte en un acompañante, guía y coequipero que aporta su experiencia y que también pone a disposición del proceso su propia voluntad de aprendizaje [10] ya que normalmente la escuela (...) no reconocía cabalmente a la persona que educaba y no encontraba alternativas eficientes orientadas a la educación y la autonomía, el diálogo, la tolerancia, la justicia y la solidaridad, lo cual representa un punto de quiebre con la escuela netamente disciplinaria [11].

La motivación forma parte de una de las tres componentes que conforman la base del aprendizaje significativo como es la actitud del estudiante que, adicional a dicha motivación, debe desarrollar capacidades para establecer nexos entre el conocimiento previo (o ya adquirido) y el nuevo conocimiento de forma que se puedan confrontar logrando que la base cognitiva se transforme [12]. Los otros dos grandes componentes de la teoría de aprendizaje significativo corresponden al conocimiento previo y el nuevo conocimiento.

El conocimiento previo está conformado por toda la herencia cognitiva que queda en el estudiante luego de su interacción permanente con los tres contextos en los cuales está inmerso: el contexto del aula, el contexto institucional y el contexto extra institucional en el cual se encuentra toda la herencia cultural que se deriva de la relación con amigos, familiares, vecinos y grupos sociales con los cuales intercambia opiniones y sentidos [13]. En la actualidad, los medios de comunicación ejercen una muy fuerte influencia en la formación de opinión de los estudiantes así como los servicios que proveen las nuevas tecnologías de la información y la comunicación [14] tales como consulta de información a través de navegadores, comunicación por WhatsApp, Twitter y correo electrónico y acceso a foros de discusión.

Sobre esta base debe advertirse que la motivación es también el elemento que dinamiza la relación entre el estudiante y lo insólito o fascinante en donde la expectativa por el aprendizaje, por lo nuevo, se compensa con los esfuerzo para adquirir nuevos conocimientos cuando se encuentra en un proceso de aprendizaje y que debe estar inscrito en un marco coherente de premios y castigos, de logros y frustraciones motivantes [15]. Lo que el estudiante descubre queda ubicado en la memoria a largo plazo y es la base para que otros aprendizajes sucedan puesto que es el resultado de las relaciones posibles que puede establecer el cerebro entre la gran cantidad de datos, información y conocimiento que acumula en la memoria y que, apropiadamente combinados, puede considerarse como experiencia.

En este sentido vale la pena recordar que tanto el descubrimiento como el significado son elementos que se 
derivan de la participación apropiada de un cerebro en un proceso de aprendizaje [16] y que dicha interacción es la que posibilita la asimilación del conocimiento para que posteriormente se pueda aplicar, retroalimentar o validar en situaciones similares o diferentes. Para ello debe tenerse en cuenta que el cerebro, de manera autónoma, ubica la información y el conocimiento que se utiliza con más frecuencia, en la memoria a largo plazo y de manera análoga aquella información que usa muy poco la ubica en la memoria a corto plazo al punto que si se trata de información que posiblemente nunca se use, entonces el mismo cerebro la desecha [17]. De allí que el gran reto del docente radica en lograr que el estudiante aprenda a largo plazo aquellos conocimientos que le serán útiles más adelante y en cuya permanencia tendrá gran importancia la motivación que haya tenido y el camino recorrido para adquirirlos.

Son precisamente estos los elementos de juicio sobre los cuales se fundamenta la estrategia Brain Based Learning, es decir, aprendizaje basado en el cerebro que, junto con otros conceptos propios de la fisiología y funcionamiento del cerebro humano, permiten que un proceso de aprendizaje que tenga en cuenta las características del cerebro tiende a ser mucho más exitoso que aquel que no las tenga en cuenta [18]. El aprendizaje basado en el cerebro considera no solo la adquisición del conocimiento disciplinar específico y el camino para acceder a él sino también las relaciones posibles que tiene el ser humano con su entorno y consigo mismo y la manera como estas relaciones influyen en dicho proceso de aprendizaje [19] que, por momentos, puede motivarlo y en otros momentos puede inhibirlo.

De esta forma, la metodología explicada y adoptada en este artículo busca aprovechar estas características para alcanzar los objetivos de aprendizaje en el área de la programación de computadores por un camino más simple, más efectivo y más breve [20] de forma que sea posible acceder cada vez a un mayor volumen de conocimiento. Lo que más se aprovecha en esta metodología es que cuando el cerebro se encuentra en situaciones de tensión, lo que suceda quedará automáticamente grabado en la memoria a largo plazo [21]. El detalle de este concepto se explicará mucho mejor en la sección de Metodología.

\section{METODOLOGÍA}

Para el desarrollo de la investigación que inspira el presente artículo se procedió metodológicamente de la siguiente forma: cada grupo de programación asignado se dividió en 2 subgrupos escogiendo los estudiantes de manera puramente aleatoria teniendo en cuenta que son estudiantes cuyas edades oscilan entre los 16 y los 20 años, todos de I semestre universitario que hasta hace poco aún estaban en el colegio, con algunos principios de programación pero sin experiencia amplia en esta área de conocimiento. Con uno de los subgrupos se aplicó la estrategia motivacional orientada (en adelante EMO) durante la realización de las pruebas escritas parciales a lo largo del semestre. Con el otro subgrupo se realizaron las pruebas escritas de la manera tradicional, es decir, sin estrategias motivacionales orientadas. Durante el semestre se realizaron tres pruebas parciales escritas, cada una con un peso del $20 \%$ sobre la nota total. Al finalizar el semestre, se realizó un examen final con un peso del $30 \%$. El 10\% restante se asumió como una nota apreciativa en la cual a todos los estudiantes se les asignó un valor 5.0 a manera de motivación. Con todos los subgrupos, se aplicaron como estrategia del proceso de aprendizaje de la programación tanto la exposición magistral como el desarrollo de ejercicios, la resolución de dudas y las consultas dentro y fuera de la clase. A todos los estudiantes se les impartió el conocimiento procurando mantener los mismos criterios de tiempo, modo y lugar para poder conferirle a los resultados un nivel de objetividad mayor.

Las estrategias motivacionales orientadas se utilizaron solamente en las pruebas parciales. El examen final fue presentado por todos los estudiantes bajo los parámetros de la metodología tradicional, es decir, sin ningún tipo de estrategia motivacional. El objetivo era comparar los resultados cuantitativos de los exámenes finales y, sobre esos resultados, hacer los análisis respectivos. A todos los estudiantes se les informó de los objetivos de la investigación, de sus características y de la metodología que se usaría para tal fin. En ninguna de las pruebas se les informó a los estudiantes previamente cuáles iban a ser las estrategias motivacionales orientadas y solamente en el momento de ser usadas, se plantearon, se cronometraron y se hicieron efectivas.

Durante las pruebas parciales, con el subgrupo que se seleccionó para aplicar la metodología propuesta, se les informó en cualquier momento, de manera repentina, que podían realizar, por uno o dos minutos cronometrados, una de tres posibles acciones (en cada oportunidad se especificaba la acción que podían realizar): a) preguntarle o discutir con un compañero, b) consultar en su propio cuaderno o en alguno de los libros que tenga en el momento (sin opción de pedir prestado ni de prestar libros) c) consultar por Internet usando su aparato celular o un computador portátil si fuere el caso. Se estableció el lapso de 1 o 2 minutos dado que es un tiempo que aumenta la tensión en medio de una prueba escrita y le posibilita al estudiante una consulta ágil, siempre y cuando él conozca lo que quiere buscar. Cada acción se llegó a utilizar sólo una vez durante una misma prueba y se procedió de manera rigurosa con el control del tiempo en su uso. Entre una acción y otra se cronometraron en promedio entre 15 y 20 minutos con el ánimo de que el cerebro alcanzare un mínimo de nivel de tensión requerido para promover el aprendizaje. Nunca se dejó realizar la acción por un tiempo superior a 2 minutos pues lo que se pretendía era aprovechar la tensión natural que se siente cuando se está en medio de la presentación de una prueba escrita.

La estrategia que se aplicó, en términos resumidos, consiste en lo siguiente:

- Se prepara una prueba escrita que esté en sintonía con la temática y los objetivos de aprendizaje que se quieren lograr dentro del marco del área de conocimiento específico, articulándolo con situaciones de algunos de los contextos propios (aprendizaje significativo) 
- Para el caso de la programación de computadores y de otras asignaturas propias de las ciencias básicas, se plantean situaciones problémicas que tengan un nivel de dificultad que puedan ser resueltas por los estudiantes de manera que la prueba escrita esté dentro de un marco coherente de premios y castigos (aprendizaje por descubrimiento)

- El día y hora de la prueba escrita se establecen las condiciones que el docente considere pertinentes para el desarrollo y resolución de la prueba escrita sin excluir a) hacer hincapié en que las pruebas son individuales, b) no acudir a ningún tipo de ayuda tecnológica, c) no acudir a ningún otro tipo de ayuda (cuadernos, libros, etc), d) no prestarse absolutamente nada entre compañeros

- Se intenta plantear un ambiente cómodo para el desarrollo de la prueba escrita sin desconocer que, por su misma naturaleza, este tipo de situaciones traen cierta tensión consigo mismas (Brain based learning)

- Luego de que los estudiantes han leído los enunciados y el ambiente de tensión propio de una prueba escrita se ha elevado a los niveles naturales, pasada alrededor una hora después de iniciada, se les anuncia que, por dos minutos cronometrados, pueden consultar en cuadernos y libros que tengan disponibles, estableciendo claramente que no pueden prestarse ni compartir ninguno de estos elementos (Brain based learning, características del cerebro)

- Pasados estos dos minutos y después de que la prueba escrita ha entrado en su dinámica individual normal, transcurridos aproximadamente 15 minutos, se les anuncia que pueden discutir y/o consultarle a algún compañero (Brain based learning, características del cerebro) por 2 minutos cronometrados (Brain based learning, características del cerebro)

- Después de estos otros dos minutos de consulta y/o discusión con un compañero, y pasados otros 15 minutos, se les indica que pueden consultar en algún dispositivo electrónico que tengan a la mano (Tablet, celular, portátil) en un tiempo cronometrado de 2 minutos (Brain based learning, características del cerebro)

- Terminada esta tercera opción de consulta, restan 15 minutos para que el estudiante entregue la prueba escrita.

Es de anotar que esta estrategia se aplica solamente con el subgrupo seleccionado para tal fin. El otro subgrupo sigue dentro de la forma tradicional de desarrollo de pruebas escritas (individual y $\sin$ opciones de consulta ni socialización). Igualmente la razón para cronometrar los minutos de consultas y socialización, preferiblemente en voz alta, radica en que eso obliga a los estudiantes, y directamente a sus cerebros, a intentar precisas cuál es la duda que tienen y no limitarse solamente a copiar.

Se tomaron resultados cuantitativos de las pruebas parciales, se tabularon dichos resultados y se organizaron para que, a partir de una presentación apropiada, se pudieran realizar las inferencias que correspondieran. Precisamente los resultados obtenidos se presentan a continuación y en ellos se compara lo que se obtuvo en las pruebas parciales con los subgrupos en los cuales se utilizaron las estrategias motivadoras orientadas y los resultados obtenidos con los subgrupos que se evaluaron por el método tradicional. De la misma manera se presentan los resultados del examen final que es el punto evaluativo en donde convergen los posibles efectos de la metodología utilizada en las pruebas parciales. Para facilitar los análisis respectivos se promediaron las notas obtenidas por los estudiantes en las diferentes pruebas escritas, grupo por grupo.

\section{RESUltados}

La tabla I presenta los resultados obtenidos en las pruebas parciales por los estudiantes con los cuales se aplicaron las estrategias motivadoras orientadas. Es de anotar que en las pruebas parciales escritas, el texto de los enunciados y de los ejercicios difería solamente en los elementos cuantitativos que se involucraban y en la distribución textual, mas no en el fondo, pues se trataba de que dichos textos problema fueran lo más similares posibles. Los enunciados eran contextuales, es decir, con alta cercanía con la realidad que vive el estudiante a diario. Esto permite aplicar el aprendizaje significativo y el aprendizaje por descubrimiento en toda su magnitud pues se le confiere significado a las situaciones problema y además se le permite al alumno encontrar soluciones a situaciones que podrían ser familiares. La aplicación de estas teorías posibilita que se pueda acudir al BBL (Brain Based Learning) en unión con las nuevas tecnologías para que se capitalicen las estrategias motivadoras orientadas en pro del aprendizaje de la programación.

TABLA I.

RESULTADOS - SUBGRUPOS CON EMO*

\begin{tabular}{|c|c|c|c|c|}
\hline Año & Sem & $\begin{array}{c}\text { Parcial } \\
1\end{array}$ & $\begin{array}{c}\text { Parcial } \\
2\end{array}$ & $\begin{array}{c}\text { Parcial } \\
3\end{array}$ \\
\hline \multirow[b]{2}{*}{2016} & I & 4,3 & 4,5 & 4,2 \\
\hline & II & 4,1 & 4,0 & 4,1 \\
\hline \multirow{2}{*}{2017} & I & 4,2 & 4,2 & 4,5 \\
\hline & II & 4,3 & 4,1 & 4,3 \\
\hline \multirow{2}{*}{2018} & I & 4,4 & 4,1 & 4,2 \\
\hline & II & 4,2 & 4,3 & 4,2 \\
\hline \multicolumn{2}{|c|}{ Prom de prom } & 4,3 & 4,2 & 4,3 \\
\hline
\end{tabular}

En la tabla II se presenta los resultados equivalentes, es decir, los que obtuvieron en las pruebas parciales los estudiantes con los cuales no se aplicó ninguna estrategia motivadora orientada.

TABLA II. RESULTADOS - SUBGRUPO SIN EMO

\begin{tabular}{lcccc}
\hline \hline \multirow{2}{*}{ Año } & Sem & Parcial & Parcial & Parcial \\
& & 1 & 2 & 3 \\
2016 & I & 3,6 & 3,5 & 3,3 \\
& II & 3,4 & 3,5 & 3.6 \\
2017 & I & 3,5 & 3,4 & 3,4 \\
& II & 3,5 & 3,8 & 3,4 \\
2018 & I & 3,4 & 3,3 & 3,3 \\
\multirow{2}{*}{ Prom de prom } & 3,5 & 3,4 & 3,2 \\
\hline \hline \multicolumn{5}{c}{ Fuente: Elaboración propia } \\
\hline \multicolumn{5}{c}{}
\end{tabular}

Fuente: Elaboración propia 
En la tabla III se presentan los resultados obtenido por cada subgrupo en el respectivo examen final para que, de manera comparativa, se realicen los análisis a que haya lugar.

TABLA III EXAMEN FINAL - AMBOS SUBGRUPOS

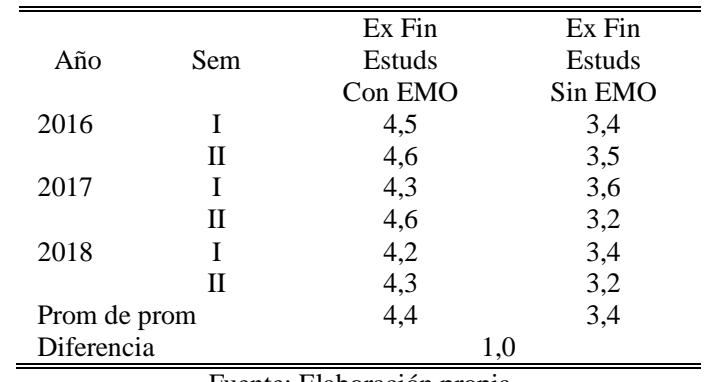

Fuente: Elaboración propia

\section{DISCUSION}

En primer lugar debe admitirse que los criterios de reagrupamiento de los cursos, por ser aleatorios de manera absoluta, podrían llegar a tener algún tipo de sesgo pero precisamente esa es parte de la metodología que le dejamos a los resultados para que éstos sean analizados posteriormente. A partir de los elementos de juicio que provee la investigación educativa se considera que la metodología adoptada para realizar el presente estudio es apropiada por cinco razones específicas: a) se acude a los protagonistas del proceso de aprendizaje como son los mismos estudiantes, b) la investigación se realiza dividiendo cada grupo en dos subgrupos que no se traslapan y que se pueden controlar en cuanto a las estrategias motivadoras orientas que se utilizan con uno y no con otro, c) se recogen todos los resultados cuantitativos posibles en relación con la investigación y que se derivan de tres pruebas parciales y un examen final, d) se tabulan estos resultados acudiendo al promedio en cada curso para poder realizar inferencias comparativas y e) se realiza todo el proceso a lo largo de seis semestres, tiempo suficientemente prudencial para analizar y determinar las tendencias en la investigación que permitan realizar inferencias acerca del proceso de aprendizaje de la programación de computadores así como que permitan extrapolarlas hacia otras áreas en donde se quiera, y sea posible, acoger las estrategias que se han explicado en este artículo. Posiblemente un análisis de la aplicación de estas estrategias en otras áreas de conocimiento permita enriquecer las conclusiones que se han obtenido y que se exponen en este artículo.

Si se analizan los resultados presentados tanto en la tabla I como en la tabla II se puede observar que, en todo momento, las notas de las pruebas escritas parciales en los subgrupos en los cuales se adoptaron las estrategias motivadoras orientadas son superiores que los grupos en donde estas estrategias no se utilizaron. El promedio de los promedios de las notas en la tabla I, en cada prueba parcial, es superior a cada una de las notas equivalentes que se presentan en la tabla II. De esta forma, la terna [4.3, 4.2, 4,3] en la última fila de la tabla corresponde al promedio de las notas de las evaluaciones parciales de los estudiantes con los cuales se adoptaron las estrategias motivadoras orientadas y la terna $[3.5,3.5,3.3]$ corresponde al promedio de las notas equivalentes obtenidas por los estudiantes que presentaron las pruebas parciales por el método tradicional, es decir, sin uso de estrategias motivadoras orientadas.

El punto en donde los dos subgrupos convergen en igualdad de condiciones es el examen final que, según se muestran los datos de la tabla III, presenta una diferencia promedio de una unidad completa $(1,0)$ entre el promedio de promedios del examen final de los subgrupos con EMO y su equivalente de los subgrupos sin EMO. El hecho de que el promedio de promedios del examen final sea más alto en estudiantes con EMO, considerando que siempre se realizó el mismo examen final a los dos subgrupos de cada grupo de programación, indica que el efecto de que a los estudiantes se les concedan las franquicias metodológicas que se han explicado en momentos de alta tensión académica es que lo que aprenden en esos micro instantes queda ubicado en la memoria a largo plazo, toda vez que el contenido de los exámenes finales siempre incluyó todos los temas vistos a lo largo de las 16 semanas que conforman el semestre académico.

Por esta razón, y tomando como punto de partida tanto los resultados puramente cuantitativos, la observación del avance de los alumnos por parte del profesor y el aumento del nivel progresivo de las inquietudes que formulan a lo largo del proceso de aprendizaje, puede concluirse que efectivamente aprendieron a programar refiriéndose con ello a la apropiación de los niveles básicos de programación funcional, sus conceptos asociados (estrategia divide y vencerás, atomización de una solución, recursividad y manejo de conjuntos de datos) y su aplicación en la construcción de una solución.

\section{CONCLUSIONES}

Con los resultados obtenidos en la presente investigación, con su análisis y los efectos en el desarrollo de los exámenes finales se concluye que todo aquello que el ser humano aprende en momentos de tensión académica, como la presentación de una prueba parcial, tiende a ubicarse en la memoria a largo plazo y por lo tanto podría considerarse como conocimiento aprendido que podría coincidir con los objetivos de aprendizaje planteados en una determinada asignatura que, en este caso, es programación de computadores.

De otra parte, la apropiación del conocimiento le exige al docente, independiente del área específica en donde se desempeñe pero más aún a aquellos docentes que no provienen de áreas con formación en educación, que conozca, aplique, evalúe, experimente, retroalimente y valide teorías, modelos y estrategias que pueden ser de gran utilidad en el desarrollo de su participación como docente pues posibilitaría resultados más efectivos y duraderos con un esfuerzo menor.

De acuerdo a la experiencia, intentar comprender y articularse con el pensamiento, los contextos de aprendizaje y las 
diferentes emociones que se involucran en los procesos de aprendizaje de los jóvenes de hoy constituye un elemento clave en el logro de los objetivos que se trazan dentro del marco de un ciclo de formación, sea este básico o superior. Investigar en educación implica arriesgar un poco para poder avanzar en el logro de los objetivos de aprendizaje y, adicional, poder entender el pensamiento de una juventud que es la que, en estos momentos, se está formando.

Se concluye entonces que, sobre una base científica concreta y apoyada en los elementos de juicio que la conformen, vale la pena aprovechar los momentos de alta tensión académica para que se fortalezca el aprendizaje $\mathrm{y}$, ante todo, para que el estudiante aprenda aprovechando características naturales del cerebro.

\section{REFERENCIAS}

[1] D. Ausubel. Sicología Educativa: Un enfoque cognitivo.New York: McGraw Hill, 2010.

[2] W. Herrmann. The whole brain bussiness book. New York: McGraw Hill. 2015.

[3] A. Davis, "The credentials of brain based learning", Journal of Philosophy of Education, vol 38, No.1, pp.21. 2014. DOI: 03098249.2004.00361.

[4] J. De Zubiría Samper, "El papel de la investigación en la consolidación de las innovaciones", Revista Educación y Ciudad (IDEP), Vol. 32, pp 15-21. Junio 2017. DOI: 10.36737/01230425.v0.n32.2017.1623.

[5] M. Mejía, "La innovación: Asunto central del siglo XXI. Una búsqueda educativa para modernizar - transformar la escuela", Revista Educación y Ciudad, Vol. 32, pp. 23-41, Junio 2017. DOI: 10.36737/01230425.v0.n32.2017.1626.

[6] A. Araya. "La educación como desarrollo personal: propuesta de innovación educativa", Revista Educación y Ciudad, Vol. 32, pp. 43-51, Junio 2017. DOI: $10.36737 / 01230425 . v 0 . n 32.2017 .1625$

[7] O.I. Trejos, "WhatsApp como herramienta de apoyo al proceso de enseñanza y aprendizaje de la programación de computadores", Revista Educación y Ciudad, Vol 35, pp. 149-158, Diciembre de 2018. DOI: 10.36737/01230425.v0.n35.2018.1970.

[8] J. Medina, Los 12 principios del cerebro. Bogotá: Grupo Editorial Norma, 2010.

[9] D. Ausubel. The Acquisition and Retention of Knowledge. Washington: Springer, 2012.

[10] F. Diaz-Barriga, F., \& G. Hernandez. Estrategias docentes para un aprendizaje significativo. México: McGraw Hill, 2002.

[11] R. Cortés, "Ser responsables de sí mismos: Las políticas educativas de los estándares, las competencias y los talentos", Revista Educación y Sociedad (IDEP), Vol. 34, pp. 77-88, Junio 2018. DOI: 10.36737/01230425.v0.n34.2018.1874.

[12] J. Bruner. Hacia una teoría de la instrucción. México: Editorial Limusa. 2006.

[13] S. Aamodt. \& S. Wang. Entra en tu cerebro. Barcelona: EdicionesB, 2008.

[14] J. Martin-Barbero. De los medios a las mediaciones: Comunicación, cultura y hegemonía. Barcelona: Anthropoos Editorial, 2010.

[15] J. Bruner. Actos de Significado. Madrid: Alianza Editorial, 2009.

[16] F. Manes \& M. Niro. Usar el cerebro. Bogotá: Grupo Editorial Planeta, 2014.

[17] O.I. Trejos. Significado y Competencias. Pereira (Risaralda) - Colombia: Editorial Papiro, 2012.

[18] G. Small. Digital Brain. Barcelona: Urano, 2009

[19] E. Jensen. The learning brain. New York: Brain Store Inc, 1994

[20] O.I. Trejos. Lógica de Programación. Bogotá: Ediciones de la U, 2017.

[21] A. Ballester. Meaningful Learning in practice. Islas Canarias: Universitat de les Illes Ballears, 2011.

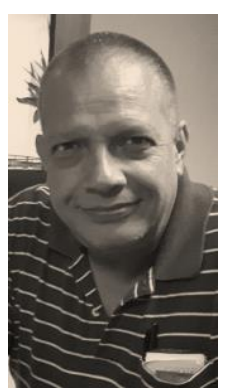

Trejos Buriticá, Omar Iván. Ingeniero de Sistemas. Especialista en Instrumentación Física. MSc en Comunicación Educativa. PhD en Ciencias de la Educación. Docente de planta categoría Titular, Programa Ingeniería de Sistemas y Computación, Facultad de Ingenierías, Universidad Tecnológica de Pereira. Autor de varios libros de programación y de una buena cantidad de artículos de investigación científica educativa en el área de la programación de computadores sobre aproximación a la optimización de procesos de enseñanza y aprendizaje dentro del contexto de la formación de ingenieros con perfil tecnológico.

ORCID: http://orcid.org/0000-0002-3751-6014 